
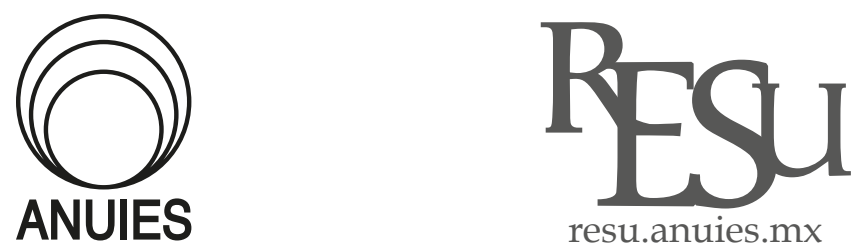

REVITA DEL
EDICACIÓN
SUPERIOR

ARTíCULO

\title{
Retención en la educación universitaria en Chile. Aplicación de un modelo de ecuaciones estructurales
}

\section{Retention in university education in Chile. Application of a structural equation model}

\author{
María Constanza Ayala Reyes* e Iván José Atencio Abarca** \\ ** Servicio de Información de Educación Superior (SIES) del Ministerio de Educación de la República de Chile. \\ Correo electrónico: van.atencio@mineduc.cl \\ Recibido el 23 de febrero del 2017; aceptado el 29 de junio del 2018.
}

\section{Resumen}

Este estudio tiene como objetivo analizar las trayectorias educativas en relación a la retención de los estudiantes universitarios chilenos, proponiendo un modelo explicativo con factores familiares, académico-educativos y económicos. Además, se analizó la variabilidad del modelo utilizando datos de estudiantes de las áreas de la salud y la educación. El modelo incorporó las propuestas de autores como Tinto y Bean, incluyendo otras experiencias tanto nacionales como internacionales. Se concluye que existen diferentes trayectorias posibles para la permanencia de los jóvenes en la educación superior, en donde los orígenes socio-económicos son determinantes debido a los altos niveles de desigualdad predominantes en la sociedad chilena.

Palabras clave: Retención, Deserción, Educación universitaria y Modelo de ecuaciones estructurales 


\begin{abstract}
The aim of this study was to analyze the educational trajectories in relation to the retention of Chilean university students, proposing an explanatory model with familiar, academiceducational and economic factors. In addition, we sought to analyze the model variability with students from health and educational areas. The model incorporated Tinto and Bean proposals, including national and international experiences. We concluded that there are different trajectories on the permanence of young people in higher education, where the socio-economic origins interact with the high levels of inequality in Chilean society.
\end{abstract}

Keywords: Retention, Dropout, University education y Structural equation model

\title{
Introducción
}

$\mathrm{E}$ conocido que uno de los cambios más significativos de los últimos treinta años en la educación superior chilena es el aumento de la matrícula, relacionada, a su vez, con el aumento de la oferta de instituciones y programas en la educación privada. Esta situación ha generado un ingreso masivo y heterogéneo de estudiantes, destacando aquellos provenientes de orígenes socialmente más vulnerables, en quienes la educación superior representa un estatuto de mejora en sus expectativas de futuro, especialmente en lo referido a los retornos económicos (Meller, 2010). A pesar de esto, en los últimos años se ha discutido fuertemente la capacidad de movilidad social asociada al paso por la educación superior, considerando nuevos dilemas en la equidad, en torno al endeudamiento, la deserción y la heterogénea calidad de las instituciones de educación superior (Orellana, 2011).

En tal sentido, las trayectorias educativas de los estudiantes se relacionan en distinta medida con determinados factores que las afectan, teniendo en cuenta los contextos social, educativo, familiar, económico, entre otros. Estas circunstancias o factores que influyen en el término exitoso de un programa o carrera son el motivo de este estudio, tanto las que determinan el abandono del ciclo, como aquellas que lo favorecen. De acuerdo con ello, la categoría central de esta investigación es la retención en la educación superior, enten- 
diéndose como la persistencia de los estudiantes en un programa de estudios de nivel terciario, hasta lograr su grado o título (Himmel, 2002).

Según datos del Servicio de Información de Educación Superior del Ministerio de Educación chileno, la retención de primer año para programas de pregrado alcanza un $70.3 \%$ para la cohorte del 2014, elevándose, en el caso de las universidades, a un $76.6 \%$. A pesar de esto, la evolución de la retención de primer año para pregrado no presenta variaciones sustanciales entre los años 2010 a 2014, lo que permite afirmar que no se han mejorado las cifras considerando los programas implementados en todo tipo de instituciones de educación superior (SIES, 2015).

Existe un desarrollo en el estudio tanto teórico como empírico sobre la retención y la deserción, siendo uno de los más reconocidos lo planteado por Vincent Tinto $(1988,1975)$, quien afirma que los estudiantes actúan de acuerdo a la construcción de su integración social y académica, relacionándose con la membresía individual en comunidades humanas. Es John P. Bean $(1985,1980)$ quien complejiza este modelo, incorporando factores académicos, psicosociales y ambientales, los cuales estarían determinando el proceso de socialización en la institución de educación superior e influyendo en la decisión final de desertar.

El presente estudio se inserta en este contexto, con el objetivo de analizar las trayectorias educativas en relación con la retención y la deserción de los estudiantes universitarios chilenos de la cohorte de ingreso del 2013, proponiendo un modelo explicativo con factores familiares, académico-educativos y económicos. Luego de esto, se busca analizar la variabilidad del modelo, aplicándolo a un segmento de estudiantes que cursan programas de las áreas de conocimiento de la salud y de la educación. La estimación de este modelo complejo busca adecuarse al caso chileno, tomando como base lo propuesto por Tinto y Bean, pero incluyendo otras experiencias tanto nacionales como internacionales, tales como: Bordón, Canals, y Rojas (2015), Barrios (2013), Duque, Duque, y Suriñach (2013), DesJardins, Ahlburg, y McCall (1999), algunos de los cuales se revisarán críticamente a continuación.

\section{Modelos explicativos sobre deserción y retención}

La literatura ofrece diferentes enfoques de estudio, clasificados como psicológicos, sociológicos, económicos, organizacionales e integracionistas (Fonseca y García, 2016; Díaz Peralta, 2008; Donoso y Schiefelbein, 2007; Himmel, 
2002). Son alrededor de 45 años de investigación que aportan teorías, herramientas analíticas, líneas de investigación, variables, categorías usadas, alcances y deficiencias.

Los modelos explicativos con una episteme sociológica clásica son los precursores en el estudio de la deserción, aspecto desarrollado por Spady (1970), quien considera que la decisión de desertar está fuertemente influenciada por la integración social de los estudiantes, homologando este fenómeno a la teoría del suicidio de Durkheim (1989), producto de la ruptura del sujeto con la sociedad por su imposibilidad de integrarse a la misma. Además de esto, el autor incorpora otros factores, como los antecedentes familiares, potencial y desarrollo académico, desarrollo intelectual, apoyo de pares, satisfacción y compromiso institucional.

El modelo de integración y adaptación longitudinal del estudiante desarrollado por Tinto $(1988,1975)$ es uno de los más relevantes e influyentes en el estudio explicativo de la deserción. Se basa en la propuesta de Spady (1970), siguiendo el supuesto de que los individuos evitan conductas que involucran algún tipo de costo en la búsqueda de beneficios en relaciones, interacciones y estados emocionales. Es así que para Tinto, la deserción se entendería como un proceso longitudinal de interacciones entre los estudiantes y los sistemas sociales de la institución, en donde las experiencias del alumno en esos sistemas modifican continuamente sus metas y compromisos institucionales de manera que desencadenará en persistencia o en alguna forma de deserción. Por otro lado Bean (1985) incorpora en la propuesta de Tinto el modelo de rotación de empleados desarrollado por Price (1977), considerando la crítica del autor al modelo de Tinto, enfocado principalmente en la imposibilidad de aplicar análisis de vías en sus modelos y la ausencia de ciertos factores externos, tales como economía y hogar (Forsman, Linder, Moll, Fraser y Andersson, 2012). Es así que el modelo propuesto por el autor considera factores académicos, psicosociales y ambientales, los cuales estarían determinando el proceso de socialización en la institución de educación superior, el cual influiría directamente en el síndrome de deserción.

Otra línea de investigación en los estudios de retención y de deserción incorpora variables de carácter psicológico en relación con lo normativo. Fishbein y Ajzen (1975) plantean que las actitudes son un elemento central dentro del proceso de toma de decisiones, ya que éstas son aprendidas, predisponen a la acción, y tales acciones son consistentemente favorables o no respecto al objeto. En ese sentido, la construcción de comportamiento es explicado por actitudes, pero también por normas subjetivas asociadas a la conducta, ya sea para continuar o dejar la educación terciaria. Este modelo permite entender las lógicas del comportamiento en las trayectorias de los 
estudiantes en educación superior y la importancia asignada a los mecanismos subjetivos que permean sus acciones. En el presente estudio no se considera la aplicación de escalas orientadas a la medición de actitudes, no obstante ello, lo propuesto por estos autores se debe tener en consideración toda vez que en el análisis resalten resultados que consideran fuentes de error desconocidas para la presente modelación y que pudieran ser explicadas a partir del comportamiento.

Ethington (1990) profundiza el modelo propuesto previamente por Fishbein y Ajzen (1975), incorporando lo planteado por Eccles y otros (1983), considerando elementos de comportamiento de persistencia, de elección y de rendimiento. Para estos autores, el fenómeno de la deserción asume que los efectos de logros pasados y experiencias de socialización están mediados por las propias interpretaciones, influenciados por construcciones culturales y percepciones sobre uno mismo (Meece, Eccles Parsons, Kaczala, Goff y Futterman, 1982). En ese sentido, Ethington (1990) propone un modelo de vías, incorporando factores sociales en el modelo explicativo, considerando antecedentes familiares, rendimiento académico previo, estímulo y apoyo familiar, los cuales estarían determinando el autoconcepto académico, la percepción de la dificultad de los estudios y el nivel de aspiraciones.

Finalmente, cabe destacar que una de las primeras aclaraciones que ofrecen los textos es la dicotomía entre dos fenómenos vinculados, pero que expresan un estatuto distinto en el estudiante, así se presentan la deserción y la retención, que son entendidos como polares y antagónicos. Como complemento a la propuesta de Himmel (2002), la retención se entenderá como la permanencia del estudiante en una carrera o programa de estudios hasta lograr su grado o su título. En contraposición, la deserción consiste en el abandono prematuro de un programa de estudios antes de alcanzar el título o grado, y considera un tiempo suficientemente largo como para descartar la posibilidad de que el estudiante se reincorpore. Este fenómeno se puede producir de manera temporal, en donde el estudiante se matricula en otro programa de la misma u otra institución, o permanente, cuando el estudiante abandona de manera definitiva la educación superior.

\section{Técnicas estadísticas para el estudio de la deserción y retención}

Los modelos de regresión probit y logit son los más utilizados por investigadores chilenos e internacionales para el estudio de la deserción o la retención. En el caso chileno, autores como Santelices, Catalán, Horn, y Kruger 
(2013), Acuña Véliz (2012), Centro de Estudios-Mineduc (2012), tienen interesantes estudios al respecto. Asimismo, para el caso internacional, autores como Belloc, Maruotti, y Petrella (2010), Montmarquette, Mahseredjian, y Houle (2001), Smith y Naylor (2001), utilizan estos modelos. A continuación se revisarán algunas de dichas aplicaciones, que resultan relevantes para este estudio ya que revelan, para el caso chileno, cierta circunscripción del tema a un solo tipo de análisis más transversal y acotado, con bajo desarrollo o experimentación con otros modelos que incorporan elementos longitudinales y causales, entre otros aspectos. No obstante, los modelos con respuesta binaria permiten orientar otros análisis, debido a que usados de manera parcial o en conjunto con otros técnicas, entregan valiosa información para organizar, por ejemplo, estudios multivariados como el que se propone en este documento.

\section{Caso de modelo probit}

Recientemente en Chile, Bordón, Canals y Rojas (2015) estimaron los determinantes de la retención de primer año en carreras e instituciones de educación superior, con ese propósito los autores utilizan un modelo probit en que la variable de respuesta es dicotómica, desertar o no desertar, calculando las probabilidades de retención de los estudiantes, a partir de un conjunto de variables explicativas. Los modelos probit permiten la estimación del efecto marginal, es decir, el cambio en Xik sobre la probabilidad de desertar.

Dentro de los principales resultados de este estudio, se observaron algunos factores que aumentan la probabilidad de permanecer en educación superior, entre los cuales se encuentra poseer un alto ranking de rendimiento en el último año de estudios secundarios, recibir una beca o tener crédito, provenir de establecimientos técnico-profesionales, y tener mayores puntajes en la Prueba de Selección Universitaria (PSU) en Lenguaje y Comunicación, y Matemáticas. Por otro lado, los factores que disminuirían la probabilidad de retención fueron estudiar en una región distinta a la de origen, proceder de un establecimiento con un mayor porcentaje de estudiantes prioritarios, provenir de establecimientos particulares, tener mayoría de edad, acceder de inmediato a educación superior, y tener compañeros en educación superior con mejores puntajes en la Psu de Matemáticas. 


\section{Caso de modelo logit}

Aplicando un modelo logit, Barrios (2013) ha estudiado el efecto de las ayudas financieras sobre la deserción y la efectividad de su focalización en los grupos de mayor vulnerabilidad. Los modelos logit trabajan con una distribución de curva logística, en donde se estima el logaritmo natural de la razón de probabilidades (más conocidos como odds ratios).

Los principales resultados obtenidos en esta investigación indican que quienes tienen menores probabilidades de desertar corresponden a: las mujeres en comparación a los hombres; los estudiantes solteros respecto a los casados; quienes terminaron la enseñanza media con mejores notas y quienes obtienen puntajes más altos en la PSU. Por otro lado, a mayor edad del estudiante al momento de ingresar a la universidad, mayores son sus probabilidades de deserción, lo mismo sucede con los estudiantes provenientes de escuelas municipales en comparación con quienes provienen de colegios particulares subvencionados o de colegios particulares. Sobre el ingreso, para aquellos estudiantes de hogares más ricos se observaron menores probabilidades de deserción que aquellos provenientes de hogares con niveles de ingresos más bajos, pero este efecto va decreciendo a medida que los ingresos aumentan. El tener algún tipo de ayuda socioeconómica reduce las probabilidades de deserción, pero este efecto va decreciendo a medida aumenta el ingreso.

\section{Crítica a los modelos con respuesta binaria y uso de otros modelos}

Diversos autores critican la utilización de los modelos de respuesta binaria para el estudio de la deserción y la retención, considerando la naturaleza longitudinal de las variables explicativas, es decir, la necesidad de incluir variables exógenas y endógenas en el modelamiento (Ishitani y DesJardins, 2002; Bean, 1980). Ésta es una de las grandes limitaciones de los estudios explicativos realizados en Chile, la sobre-utilización de técnicas estadísticas que no logran tomar en cuenta la trayectoria de los jóvenes, considerando la recursividad de los efectos (causalidad direccionada) y discretitud de las variables.

Los estudios internacionales tienden a la utilización de técnicas de modelamiento estadístico considerando un mayor nivel de complejidad, incorporando variables endógenas y exógenas, efectos directos, indirectos y 
de interacción, incluyendo información longitudinal sobre los estudiantes. Esto es lo realizado por Duque, Duque y Suriñach (2013) en España, quienes estudian las intenciones de deserción simultáneamente con los resultados del aprendizaje, utilizando modelamiento con ecuaciones estructurales, se midieron distintos constructos complejos, como la calidad de educación, calidad de recursos educacionales, confirmación de expectativas percibidas, agotamiento emocional, involucramiento estudiantil, resultados cognitivos, satisfacción estudiantil general, intenciones de deserción y resultados afectivos. Los autores concluyen que a medida que aumenta la calidad de los recursos educacionales percibidos por los estudiantes y sus expectativas previas son confirmadas, se incrementan sus resultados cognitivos y niveles de satisfacción. Por ende, la intención de deserción por parte de los estudiantes aumenta cuando no está satisfecho con su experiencia universitaria.

DesJardins, Ahlburg y McCall (1999) aplican un modelamiento estadístico de la deserción en algunas universidades de Estados Unidos, por medio de la técnica event history analysis, parte de la familia de los análisis de supervivencia, que permite controlar a través de la heterogeneidad no observada e incorporar coeficientes de variabilidad en el tiempo y otorga tolerancia para que eventos competitivos puedan ser modelados simultáneamente. Esto les permitió evaluar el peso de las variables explicativas en distintos momentos de la trayectoria de los estudiantes, considerando variables académicas previas al ingreso a la universidad, información demográfica, logros actuales, variables institucionales y financieras. Los autores observaron que, a partir de los datos, las mujeres tienen menores probabilidades de deserción en los primeros años, pero más probabilidades que los hombres de dejar más tarde sus estudios, a mayor edad al matricularse, mayores probabilidades de desertar al primer año; entre el primer y tercer año los estudiantes que se matriculan en instituciones de la misma zona geográfica tienen menores probabilidades de desertar que los que provienen de fuera; los estudiantes con más altos rankings en educación secundaria tienen menores probabilidades de desertar en los años 2, 3 y 5 hasta 7; quienes estudiaron alguna carrera antes, sin terminarla, tienen menos probabilidades de desertar en el año dos, comparado con los estudiantes que acceden por primera vez al sistema; las notas medias de educación superior tienen una relación negativa con la deserción, pero este efecto disminuye a medida pasan los años, los créditos en general reducen la probabilidad de desertar en el año 3 y del año 5 al 7; en el caso de las becas, reducen la probabilidad en el año 3.

A partir de los estudios revisados, se ha planteado como hipótesis principal de estudio la existencia de una influencia causal de la trayectoria bio- 
gráfica del estudiante (longitudinal) respecto de la retención, en donde los antecedentes familiares, económicos y académico-educativos operan como factores interrelacionados en la construcción del fenómeno estudiado. Resulta relevante indicar que, se espera que las diferencias en los orígenes socioeconómicos de los estudiantes, determinen de manera importante la permanencia en la educación terciaria.

\section{Metodología del estudio}

\section{Descripción de los datos usados}

La base de datos inicial que se utilizó para la presente investigación correspondió a la matrícula nacional en educación superior del año 2013, información recolectada por el Servicio de Información de Educación Superior (SIES). En esta base se seleccionaron a nuevos entrantes universitarios entre 17 a 25 años, ${ }^{1}$ matriculados en carreras profesionales en planes regulares de pregrado. En el caso de que estos cursaran programas sin licenciatura previa la duración de éste debía ser mayor o igual a 6 semestres, y para quienes estaban matriculados en programas con licenciatura previa, la duración debía ser mayor o igual a 8 semestres.

Esta base de datos fue complementada con información proveniente del Sistema de Información General de Estudiantes (SIGE), desde donde se obtuvo información respecto a la educación secundaria de los jóvenes. Asimismo, se utilizó la base del Departamento de Evaluación, Medición y Registro Educacional (DEMRE) de la Universidad de Chile, con información socioeconómica asociada a la PSU.

Para determinar a los estudiantes desertores y retenidos, se cruzó la base de datos con la matrícula del 2014 y 2015, en donde interesaba que los estudiantes estuviesen retenidos en la misma institución. A partir de este ejercicio se logró segmentar a los desertores en dos grupos, temporales y definitivos, los primeros corresponden a los estudiantes que en el 2015 volvieron a estudiar en la misma institución y los definitivos corresponden a los que no regresaron a la institución. Los estudiantes desertores temporales fueron agrupados como retenidos, considerando que volvieron a ingresar a la misma institución.

\footnotetext{
${ }^{1}$ Estudiantes que están por primera vez en educación superior.
} 
Considerando las precedentes condiciones, en la Tabla 1 se presenta la distribución de los casos seleccionados, bajo la condición de deserción y de retención de los estudiantes, con un total de 82.049 estudiantes, de los cuales el $67 \%$ se mantuvo en el sistema y el 33\% fueron clasificados como desertores definitivos.

Tabla 1

Distribución de la condición de deserción y retención de los estudiantes de la cohorte 2013

\begin{tabular}{lcc}
\hline Condición & Frecuencia & $\%$ \\
\hline Desertor & 27.112 & 33 \\
\hline Retenido & 54.937 & 67 \\
\hline Total & $\mathbf{8 2 . 0 4 9}$ & $\mathbf{1 0 0}$ \\
\hline
\end{tabular}

Las variables independientes corresponden a predictores exógenos y endógenos sobre características sociodemográficas y antecedentes familiares, académico-educativos y económicos de los estudiantes. Por las razones indicadas más adelante en el análisis de los resultados de las variables sociodemográficas se seleccionó el sexo y la edad; de los factores familiares se incorporó el nivel educacional de los padres, la situación laboral de la madre y la presencia de algún familiar en educación superior; respecto a variables académico-educativos, se eligieron la dependencia del establecimiento de origen y la clasificación de la institución de educación superior; por último, para la información económica se utilizaron los ingresos familiares y la presencia de algún beneficio económico. Se consideraron variables con medida nominal, ordinal y escalar (continuas), las cuales se presentan en las tablas 2, 3 y 4 respectivamente.

Tabla 2

Distribución variables independientes nominales

\begin{tabular}{llrc}
\hline Código & Nombre variable y categorías & $\mathbf{n}$ & $\%$ \\
\hline & Sexo estudiante & & \\
\hline 0 & Hombre & 37.531 & 45,7 \\
\hline 1 & Mujer & 44.518 & 54,3 \\
\hline & Perdidos & 0 & 0 \\
\hline & Tipo dependencia establecimiento origen & & \\
\hline 0 & Municipal & 19.277 & 23,5 \\
\hline 1 & Part. Subvencionado & 46.776 & 57,0 \\
\hline 2 & Particular Pagado & 14.884 & 18,1 \\
\hline & Perdidos & 1.112 & 1,4 \\
\hline
\end{tabular}




\begin{tabular}{|c|c|c|c|}
\hline Código & Nombre variable y categorías & $\mathbf{n}$ & $\%$ \\
\hline & Situación ocupacional de la madre del postulante ${ }^{2}$ & & \\
\hline 0 & Inactivo & 33.889 & 41,3 \\
\hline \multirow[t]{3}{*}{1} & Activo & 38.765 & 47,2 \\
\hline & Perdidos & 9.395 & 11,5 \\
\hline & Beneficio económico (beca o crédito) & & \\
\hline 0 & Sin beneficio & 27.995 & 34,1 \\
\hline \multirow[t]{3}{*}{1} & Con beneficio & 54.054 & 65,9 \\
\hline & Perdidos & 0 & 0 \\
\hline & Integrante grupo familiar estudia en educación superior & & \\
\hline 0 & Ninguno & 53.734 & 65,5 \\
\hline \multirow[t]{3}{*}{1} & Al menos 1 & 26.691 & 32,5 \\
\hline & Perdidos & 1.624 & 2,0 \\
\hline & Clasificación institución de educación superior ${ }^{3}$ & & \\
\hline 0 & Univ. docentes & 29.397 & 35,8 \\
\hline 1 & Univ. docentes con proyección en investigación & 13.514 & 16,5 \\
\hline 2 & Univ. docentes con investigación y doctorados en áreas selectivas & 20.232 & 24,7 \\
\hline \multirow[t]{2}{*}{3} & Univ. de investigación y doctorados & 18.906 & 23,0 \\
\hline & Perdidos & 0 & 0 \\
\hline
\end{tabular}

2 No se consideró para los modelos la situación ocupacional del padre, debido a la gran cantidad de casos perdidos (20\%) y la alta concentración de los datos en la categoría activo (casi el 70\%).

3 Para más información ver "Una nueva clasificación de las universidades chilenas" (Reyes y Rosso, 2013).

Tabla 3

Distribución variables independientes ordinales

\begin{tabular}{clrc}
\hline Código & \multicolumn{1}{c}{ Nombre variable y categorías } & $\mathbf{n}$ & $\%$ \\
\hline & Ingreso bruto total del grupo familiar & & \\
\hline 0 & 0 a 144.000 & 6.931 & 8,4 \\
\hline 1 & 144.001 a 288.000 & 21.019 & 25,6 \\
\hline 2 & 288.001 a 432.000 & 14.183 & 17,3 \\
\hline 3 & 432.001 a 576.000 & 8.303 & 10,1 \\
\hline 4 & 576.001 a 720.000 & 6.019 & 7,3 \\
\hline 5 & 720.001 a 864.000 & 3.702 & 4,5 \\
\hline 6 & 864.001 a 1.008 .000 & 3.955 & 4,8 \\
\hline 7 & 1.008 .001 a 1.152 .000 & 1.989 & 2,4 \\
\hline 8 & 1.152 .001 a 1.296 .000 & 1.232 & 1,5 \\
\hline 9 & 1.296 .001 a 1.440 .000 & 1.170 & 1,4 \\
\hline 10 & 1.440 .001 a 1.584 .000 & 1.247 & 1,5 \\
\hline
\end{tabular}

Continúa... 


\begin{tabular}{|c|c|c|c|}
\hline Código & Nombre variable y categorías & $\mathbf{n}$ & $\%$ \\
\hline \multirow[t]{3}{*}{11} & 1.584.001 a o más & 10.675 & 13,0 \\
\hline & Perdidos & 1.624 & 2,0 \\
\hline & Nivel educacional del padre del postulante & & \\
\hline 0 & Sin estudios superiores & 38.440 & 46,9 \\
\hline 1 & Estudios técnicos y profesionales incompletos IP-CFT & 5.167 & 6,3 \\
\hline 2 & Estudios técnicos y profesionales completos IP-CFT & 4.494 & 5,5 \\
\hline 3 & Estudios universitarios incompletos & 3.889 & 4,7 \\
\hline \multirow[t]{3}{*}{4} & Estudios universitarios completos & 16.162 & 19,7 \\
\hline & Perdidos & 13.897 & 16,9 \\
\hline & Nivel educacional de la madre del postulante & & \\
\hline 0 & Sin estudios superiores & 43.773 & 53,3 \\
\hline 1 & Estudios técnicos y profesionales incompletos IP-CFT & 7.402 & 9,0 \\
\hline 2 & Estudios técnicos y profesionales completos IP-CFT & 6.379 & 7,8 \\
\hline 3 & Estudios universitarios incompletos & 3.003 & 3,7 \\
\hline \multirow[t]{2}{*}{4} & Estudios universitarios completos & 13.872 & 16,9 \\
\hline & Perdidos & 7.620 & 9,3 \\
\hline
\end{tabular}

Tabla 4

Estadísticos descriptivos variables independientes escalares (continuas)

\begin{tabular}{|c|c|c|c|c|c|c|c|}
\hline $\begin{array}{l}\text { Edad del estudiante } \\
\text { al matricularse }\end{array}$ & Media & Mediana & $\begin{array}{c}\text { Desviación } \\
\text { estándar }\end{array}$ & Varianza & Mínimo & Máximo & Perdidos \\
\hline
\end{tabular}

Fuente: elaboración propia.

\section{Método de análisis y modelo}

La técnica de análisis utilizada para la construcción de un modelo explicativo radica en ecuaciones estructurales (en inglés Structural Equation Modeling, SEM), que estudia fenómenos multirelacionales de dependencia estimando el efecto y las relaciones entre múltiples variables que, si bien son dependientes en cierta estructura de relaciones, las mismas pueden jugar roles de independencia respecto de otras relaciones de variables (Bollen, 1989). Se suma la posibilidad de testear diferentes tipos de relaciones entre variables; incorpora y modela variables latentes (constructos complejos, con más de una variable manifiesta). 
Los modelos SEM se conforman de dos componentes o (sub)modelos. Un primer componente de medición, que busca estimar una o más variables latentes a partir de un conjunto de variables observables o medibles, a veces también llamados indicadores. Este análisis se denomina Análisis Factorial Confirmatorio (en inglés Confirmatory Factor Analysis, CFA) y estima una matriz de varianza-covarianza observada respecto a los indicadores que conforman las variables latentes (Brown, 2006). Esta técnica se utilizó en la presente investigación con el objetivo de testear ciertos constructos complejos presentes en las trayectorias de los estudiantes, considerando los antecedentes familiares, académico-educativos y económicos.

Un segundo componente refiere al modelo estructural que permite especificar las relaciones y efectos entre variables latentes y manifiestas, por medio de la estimación de regresiones en donde se considera no solo un conjunto de variables independientes como regresores de una variable dependiente, sino que además se puede asumir que uno más de esos regresores se encuentra en función de otras variables independientes (Skrondal y Rabe-Hesketh, 2005).

Como señalan variados autores, una de las principales relevancias de estos modelos es la posibilidad de hipotetizar efectos causales entre las variables, la concatenación de efectos, reciprocidad entre variables y desarrollar una representación gráfica, que facilita la comprensión del modelo (Díaz, Pardo Merino y San Martín Castellanos, 2010). Se debe tener en consideración que toda relación causal tiene que ser evaluada tanto en el sentido estadístico como en el sentido del problema estudiado. No toda relación causal estadística necesariamente tiene validez en lo real y, por tanto, se debe evaluar su pertinencia. En ese sentido es importante señalar que covariación y causalidad no son la misma cosa. Cuando se observa una alta relación (covariación) entre dos variables, no debemos necesariamente interpretarla, a priori, como una relación causal entre ambas, pueden existir otras variables que no se han considerado y potencien o atenúen esta relación.

Atendiendo a la naturaleza categórica de los datos, para la estimación de los modelos se utilizó el estimador robusto de mínimos cuadrados ponderados (WLSMV, por su denominación en inglés Weighted Least Squares Mean and Variance Adjusted). 


\section{Modelo de retención en Chile}

A continuación se presentan los resultados obtenidos en los modelos estimados, comenzando por el modelo general para estudiantes de universidades chilenas de la cohorte 2013, luego se ajustó para algunas áreas del conocimiento a saber; educación y salud. Esto con el objetivo de observar las variaciones de los efectos causales de la retención manteniendo las mismas variables del modelo general, pero en segmentos de la población estudiada.

Para la elección del modelo que se presenta se evaluaron diez modelos previamente, en donde las variables exógenas y endógenas fueron incorporadas por bloques o factores, los cuales fueron testeados de acuerdo a los estadísticos de ajuste, el efecto de cada variable en los modelos y la parsimonia en el contexto del problema estudiado. Además de los bloques, se incorporó la noción de trayectoria (longitudinal) de los estudiantes. En este proceso se incorporaron variables relacionadas con las capacidades y habilidades académicas de los estudiantes (PSU y NEM), pero no fueron estadísticamente significativas para el modelo, pues no permitieron un buen ajuste por el bajo efecto sobre retención. En ese sentido, estos factores poseen altas correlaciones con las variables académico-educacionales incorporadas en el modelo final (Pearson, 2013).

El modelo final tiene como variable dependiente el fenómeno de retención y consiste en diez variables observadas y una variable latente, que constituyen tanto el sub-modelo observado, como el sub-modelo estructural latente. En ellos las diversas variables que co-varían entre sí y que son significativas estadísticamente determinan la relación con la retención, siendo importante observar las orientaciones de la relación (orientación de la correlación o dirección de las flechas entre variables en el diagrama resultante).

Por otra parte, las pruebas de ajuste obtenidas por el modelo expresan que existe cercanía entre las covarianzas producidas y las observadas. El estadístico RMSEA tiene un valor de 0.06 , lo que implica que se estimó un modelo parsimonioso considerando una distribución $X^{2}$. Sumado a esto, los estadísticos CFI y TLI tuvieron valores de 0.94 y 0.91 respectivamente, indicando que el modelo estimado ajusta de mejor manera los datos que el modelo nulo. 


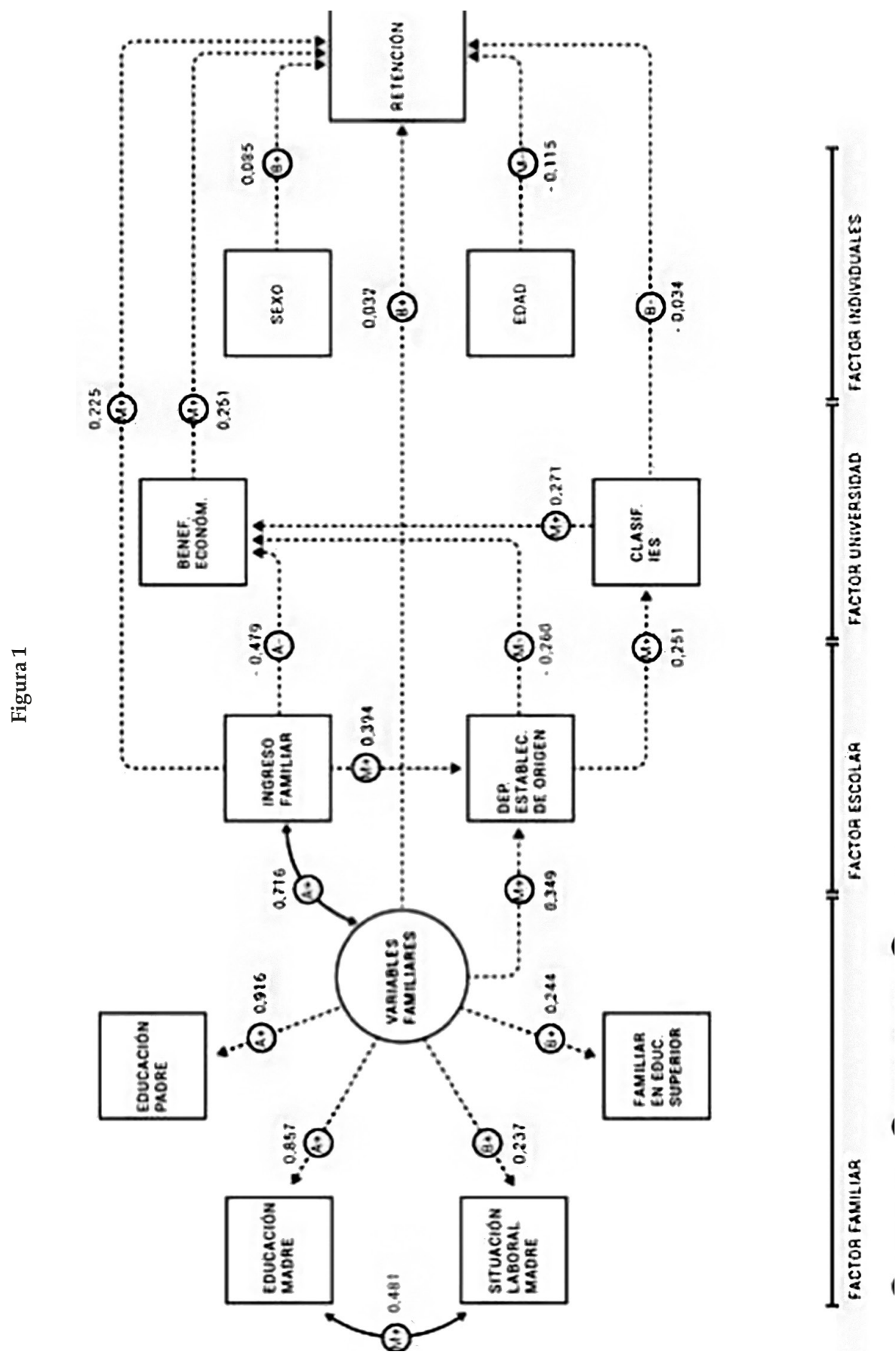


En la Figura 1 es posible observar el modelo final estimado, en donde la cercanía entre la definición teórica del modelo y la realidad del fenómeno estudiado queda en evidencia a partir de los indicadores que conforman la variable latente "antecedentes familiares", que posee una evidente connotación de parentesco con el estudiante, por medio de indicadores orientados a medir la educación del padre y madre, situación laboral de la madre y presencia de algún familiar en educación superior. Es así que las relaciones causales se pueden expresar linealmente en las siguientes ecuaciones:

(1) Educación padre $=0.916^{*}$ Familia

(2) Educación madre $=0.857^{*}$ Familia

(3) Situación laboral madre $=0.237^{*}$ Familia

(4) Familiar en educación superior $=0.244^{*}$ Familia

(5) Dependencia del establecimiento de origen $=0.349^{*}$ Familia $+0.394^{*}$ Ingreso familiar

(6) Clasificación de institución de educación superior $=0.251{ }^{*}$ Dependencia del establecimiento de origen

(7) Beneficios económicos $=-0.479 *$ Ingreso familiar $+-0.260 *$ Dependencia establecimiento de origen $+0.271^{*}$ Clasificación de institución de educación superior

(8) Retención $=0.032 *$ Familia $+0.225^{*}$ Ingreso familiar $+0.251 *$ Beneficio económico $+-0.034^{*}$ Clasificación de institución de educación superior + $0.085 *$ Sexo $+0.115^{\star}$ Edad

Los resultados de las estimaciones presentadas en el diagrama son todas significativas desde el punto de vista estadístico, esto implica que los coeficientes son distintos a cero en la población estudiada, utilizando un 99\% de confianza.

La retención tiene una relación directa con seis variables, una de las cuales refiere a los factores familiares, siendo una variable latente exógena construida a partir de cuatro variables de atributos de la familia, que poseen una relación positiva con la variable latente (ecuaciones 1-2-3-4). Así, los antecedentes familiares tienen un parámetro estimado fuerte y positivo respecto a la educación de los padres, en donde el aumento en una unidad en este constructo aumentará proporcionalmente la educación del padre (ecuación 1) o de la madre (ecuación 2), manteniendo el resto de los indicadores constante. A su vez, existen dos variables con una relación débil positiva con los antecedentes familiares, a saber, la situación laboral de la madre (ecuación 3) y la presencia de al menos un familiar en educación superior (ecuación 4). Es decir, si aumentan los antecedentes familiares del estudiante en una unidad, aumentará proporcionalmente la situación laboral de la madre o la presencia de al menos un familiar en educación superior. 
Por otra parte, los antecedentes familiares poseen una relación directa, la cual es débil y positiva, respecto a la retención. Por sí misma, los factores familiares median en la relación indirecta de sus cuatro indicadores con la retención, siendo relevante en el contexto del problema, pues aparece como un antecedente (temporal) que reúne condiciones de apoyo a la trayectoria del estudiante en la educación superior.

No obstante lo débil del efecto, una mayor educación del padre y de la madre, con una madre trabajando y al menos un familiar en educación superior, se construye un conjunto de variables de apoyo para la futura trayectoria del estudiante. Esto tiene consistencia considerando lo propuesto por expertos en el tema, expresamente lo muestran Spady (1970), Tinto (1975), Bean (1985) y Ethington (1990), que consideran a la "familia" relevante para generar condiciones propicias en el tiempo (longitudinal) previo y durante el estudio. A la vez que la "familia" corresponde a un factor externo de apoyo al logro de expectativas, adecuaciones, integración y factores económicos para la trayectoria y metas educativas del estudiante.

Los antecedentes familiares como los ingresos, tienen una relación moderada y positiva con la dependencia del establecimiento de origen (ecuación 5). Es así que se podría concluir que, a mayor educación de los padres, con una madre ocupada laboralmente, al menos un familiar en educación superior y un mayor ingreso familiar, un estudiante tiende a cursar sus estudios secundarios en un establecimiento particular pagado. Cabe destacar que entre los antecedentes familiares e ingresos familiares existe una relación recíproca alta y positiva, es decir, son directamente proporcionales entre ambos elementos.

Estos resultados se condicen con múltiples estudios y evidencias que indican que el sistema educativo chileno está fuertemente segregado, en donde quienes poseen mayor capital económico, social y cultural atienden a colegios particulares pagados, quienes tendrán mayores beneficios en el futuro, tanto referido al rendimiento académico (Pearson, 2013), acceso y permanencia en instituciones de educación superior de mayor calidad (Espinoza y González, 2013), influyendo en la posibilidad y la calidad del trabajo e ingresos (Urzúa, 2012).

Cuando se observan los resultados respecto a la clasificación de las instituciones de educación superior, se debe considerar que se produce una relación concatenada entre los antecedentes familiares e ingreso familiar que afecta en la dependencia del establecimiento de origen de los estudiantes, en tanto esta última es eventual causa de la clasificación de las instituciones de educación superior (ecuación 6). La relación entre ambas variables es directa, moderada y positiva, en donde los estudiantes que provienen de colegios 
particulares subvencionado o pagado tienden a ingresar a instituciones de educación superior más selectivas.

El cómo influye la cuestión financiera - a través de la familia - en el tipo de colegio en el cual se estudia es clave para determinar el tipo de institución de educación superior a la cual se ingresará. Los beneficios económicos están afectados por una triada de variables manifiestas, dos de las cuales tienen una relación negativa y moderada, referido a los ingresos familiares y la dependencia del establecimiento de origen, por otra parte, una relación positiva moderada con la clasificación de las instituciones de educación superior (ecuación 7). Esto se puede interpretar de la siguiente manera, un estudiante con menores ingresos familiares, proveniente de un colegio municipal y de una institución de educación superior más selectiva, tiende a acceder a beneficios económicos para proseguir sus estudios en educación superior. Estos resultados son esperables, considerando que quienes acceden a beneficios económicos provienen en su mayoría de los primeros quintiles de la población (DFE, 2015).

Cabe señalar que los beneficios económicos tienen una relación directa con la retención, y se pudiera señalar que cierra el círculo de las variables relevantes en el tema de la retención, en tanto se configura una tendencia a una interrelación familiar-institucional fuertemente ligada a la cuestión económica, que afecta decisivamente durante la trayectoria educativa del estudiante, como se señaló anteriormente.

La ecuación 7 ya constituye una primera síntesis del modelo, de tal modo que el ingreso familiar, la dependencia del establecimiento de origen (ambas se relacionan con los antecedentes familiares), y al sumar la clasificación de instituciones de educación superior, permiten apreciar más claramente la concatenación temporal o longitudinal (Tinto, 1975), además de los factores ambientales, académicos y psicosociales, en la biografía del estudiante, así como el rol que juegan las instituciones, afectan la decisión de permanencia del estudiante hasta el logro educativo esperado.

El fenómeno de la retención queda así estructurado en términos longitudinales, en donde los factores familiares, escolares y universitarios se conjugan influyendo en las posibles direcciones que tome un estudiante. Sobre los efectos directos, solamente el tipo de institución de educación superior posee una relación débil y negativa, lo que indica que mientras más compleja es la institución, la retención es menor (considerando las demás variables de la relación fijas), es decir, existen mayores obstáculos para lograr permanecer en la institución de educación superior.

En el caso de los antecedentes familiares, ingresos familiares y beneficios económicos, se presentan parámetros positivos con respecto a la retención. 
En donde si un estudiante posee becas o créditos, tiende mayormente a permanecer en la educación superior (manteniendo los otros parámetros fijos). Similar es lo que ocurre con los ingresos familiares, en donde a mayores ingresos familiares, los estudiantes tienden a permanecer en el sistema de educación superior.

Como se comentó previamente, los antecedentes familiares poseen una relación positiva, pero baja respecto a la retención, es decir, a mayor educación del padre y de la madre, con una madre trabajando y al menos un familiar en educación superior, un estudiante tiende a permanecer en la institución.

Se incluyeron dos variables de control en el modelo, sexo y edad, en el primer caso los resultados indican que las mujeres tienen mayores posibilidades de permanecer en la educación superior en comparación a los hombres, no obstante, el parámetro que relaciona sexo con retención es débil, pero positivo. Con respecto a la edad de los estudiantes, se observa que a menor edad de los estudiantes al matricularse, mayores posibilidades de retención en educación superior, teniendo en cuenta que el parámetro estimado es débil y negativo. A similares conclusiones han llegado otros estudios, en donde las mujeres poseen menores probabilidades de desertar de sus estudios universitarios, y mientras mayor es la edad de los estudiantes al momento de la matrícula, mayores son las probabilidades de desertar (Bordón, Canals y Rojas, 2015; Barrios, 2013; DesJardins, Ahlburg y McCall, 1999).

En resumen, se pueden observar cuatro rutas que facilitan la permanencia del estudiante en la institución de educación superior:

Ruta 1: Antecedentes familiares $\rightarrow$ Ingresos familiares $\rightarrow$ Retención

Ruta 2: Antecedentes familiares $\rightarrow$ Dependencia del establecimiento de origen $\rightarrow$ Beneficio económico $\rightarrow$ Retención

Ruta 3: Antecedentes familiares $\rightarrow$ Dependencia del establecimiento de origen $\rightarrow$ Clasificación de la institución de educación superior $\rightarrow$ Retención

Ruta 4: Antecedentes familiares $\rightarrow$ Dependencia del establecimiento de origen $\rightarrow$ Clasificación de la institución de educación superior $\rightarrow$ Beneficio económico $\rightarrow$ Retención

Las dos primeras rutas muestran dos tipos estudiantes, uno con mayor capital económico, en contraposición a la segunda ruta, para la cual es relevante el beneficio. La tercera ruta posee un efecto negativo respecto a la retención cuando se considera la clasificación de la institución de educación superior, es decir, hay instituciones en las cuales existen mayores exigencias para la permanencia. La última ruta incluye una mayor cantidad de variables que influyen en la trayectoria del estudiante y en su posible permanencia en el 
sistema. Esto se expresa en la bifurcación que provoca la clasificación de la institución de educación superior, en donde afecta directamente la retención o media a través de los beneficios económicos.

\section{Retención en áreas de salud y de educación}

A continuación se presentan dos modelos que fueron ajustados a las áreas del conocimiento educación y salud, con el objetivo de observar la variabilidad de los efectos de los elementos incluidos cuando se focaliza el análisis en ellos. En la tabla 5 se observan los resultados de los modelos estimados, algunos efectos fueron eliminados debido a que no presentaban resultados significativos.

Tabla 5

Modelos estimados para áreas de conocimiento de salud y educación

\begin{tabular}{|c|c|c|c|c|}
\hline & \multicolumn{2}{|c|}{ Salud } & \multicolumn{2}{|c|}{ Educación } \\
\hline & & & Coeficiente & $\begin{array}{c}\text { Desviación } \\
\text { estándar }\end{array}$ \\
\hline \multicolumn{5}{|l|}{ Antecedentes familiares por } \\
\hline Educación padre & 0,908 & 2,162 & 0,908 & 2,168 \\
\hline Educación madre & 0,851 & 1,618 & 0,816 & 1,411 \\
\hline Situación laboral madre & 0,253 & 0,262 & 0,193 & 0,196 \\
\hline Familiar en ed. superior & 0,229 & 0,235 & 0,298 & 0,312 \\
\hline \multicolumn{5}{|c|}{$\begin{array}{l}\text { Dependencia establecimiento de } \\
\text { origen en }\end{array}$} \\
\hline Antecedentes familiares & 0,350 & 0,462 & 0,271 & 0,329 \\
\hline Ingresos familiares & 0,354 & 0,142 & 0,356 & 0,158 \\
\hline \multicolumn{5}{|c|}{$\begin{array}{l}\text { Clasificación institución de ed. } \\
\text { superior en }\end{array}$} \\
\hline Dependencia est. origen & 0,200 & 0,154 & 0,152 & 0,126 \\
\hline Beneficio económico en & & & & 1,4 \\
\hline Ingresos familiares & $-0,470$ & $-0,189$ & $-0,214$ & $-0,089$ \\
\hline Dependencia est. origen & $-0,254$ & $-0,254$ & $-0,127$ & $-0,120$ \\
\hline $\begin{array}{l}\text { Clasificación institución de } \\
\text { educación superior }\end{array}$ & 0,199 & 0,257 & 0,424 & 0,480 \\
\hline \multicolumn{5}{|l|}{ Retención en } \\
\hline Antecedentes familiares & 0,066 & 0,069 & - & - \\
\hline Ingresos familiares & 0,254 & 0,081 & 0,092 & 0,035 \\
\hline Beneficio económico & 0,338 & 0,269 & 0,243 & 0,219 \\
\hline $\begin{array}{l}\text { Clasificación institución de } \\
\text { educación superior }\end{array}$ & - & - & $-0,079$ & $-0,081$ \\
\hline
\end{tabular}




\begin{tabular}{|c|c|c|c|c|}
\hline & \multicolumn{2}{|c|}{ Salud } & \multicolumn{2}{|c|}{ Educación } \\
\hline & & & Coeficiente & $\begin{array}{c}\text { Desviación } \\
\text { estándar }\end{array}$ \\
\hline Sexo & 0,037 & 0,087 & 0,086 & 0,185 \\
\hline Edad & $-0,100$ & $-0,115$ & $-0,115$ & $-0,101$ \\
\hline \multicolumn{5}{|c|}{ Antecedentes familiares con } \\
\hline Ingresos familiares & 0,717 & 2,352 & 0,631 & 1,730 \\
\hline \multicolumn{5}{|l|}{ Educación madre con } \\
\hline Situación laboral madre & 0,530 & 0,530 & 0,464 & 0,464 \\
\hline $\mathrm{X} 2(37)$ & $2.202(p=0,00)$ & & $803(p=0,00)$ & \\
\hline RMSEA & 0,05 & & 0,05 & \\
\hline CFI & 0,95 & & 0,95 & \\
\hline TLI & 0,92 & & 0,92 & \\
\hline
\end{tabular}

Respecto al área de educación, el principal hallazgo consiste en que los antecedentes familiares no tienen una relación directa con la retención, sumado a que disminuye la relación de los ingresos familiares con ella. Estos resultados se pueden interpretar a partir del hecho que los programas asociados al área de educación no se reconocen por ser mayormente selectivos, especialmente en el caso de las carreras de pedagogía (Manzi Astudillo, 2011).

En contraposición, se observa que para el área de salud, el efecto de la clasificación de la institución de educación superior es eliminado, ya que no es estadísticamente significativo. Además, tanto los antecedentes familiares como los beneficios económicos aumentaron sus parámetros, es decir, presentan mayor importancia respecto a la trayectoria de permanencia de los estudiantes en educación superior. Cabe señalar que los programas del área de salud requieren mayores recursos para lograr la permanencia de los estudiantes.

Es posible, entonces, observar una diferencia entre ambas áreas del conocimiento. Así los estudiantes que cursan programas en el área de educación no requieren de mayores recursos, además que el sistema de ingreso es más flexible. Esto se contrapone a lo que sucede con los estudiantes en programas en el área de salud, en donde es necesario poseer mayores recursos, además de que poseen procesos de selección más rigurosos. Estas diferencias se plasman también en los eventuales ingresos económicos de los titulados de estas áreas, considerando que quienes se titularon de programas del área de salud poseen ingresos sustancialmente mayores respecto a quienes se titularon del área de educación tanto al primer y quinto año de titulación (Atencio Abarca y López, 2016a, 2016b). 


\section{Conclusiones}

De acuerdo a los objetivos propuestos, se ha construido un modelo de factores que explican la trayectoria asociada a la retención de los estudiantes chilenos, utilizando la técnica de análisis estadístico de ecuaciones estructurales. Este modelo logró satisfacer las exigencias estadísticas, por una parte, a la vez que posee parsimonia y sentido respecto del tema. De acuerdo a lo planteado en la hipótesis central de trabajo, efectivamente se realizó un modelo que pudiese explicar la influencia causal de la trayectoria biográfica del estudiante respecto a la retención, incorporando factores académico-educativos y económicos respecto a la familia.

El modelo general presentado muestra la relevancia de la familia como antecedente para el ingreso del estudiante al sistema educativo y eventualmente en la permanencia en la educación superior. Es así que las cuatro covariables que construyen la variable de "antecedentes familiares", a saber, niveles de educación del padre y la madre, situación laboral de la madre, y presencia de algún familiar en la educación superior, aportan a la retención de manera directa e indirecta a la vez, por medio de los efectos que produce respecto a los ingresos familiares y la dependencia del establecimiento de origen.

Se observó cómo esta relación entre los factores familiares y retención tiene dos vías de influencia positiva, tanto a través del ingreso familiar (ruta 1), así como indirectamente a través de los beneficios económicos (ruta 2). De esta manera se comprueba que el factor económico es relevante para la retención en educación superior, considerando que las variables ligadas a lo económico aportan significativamente a la varianza explicada. Estos resultados van en la línea con lo planteado por otros autores (Spady, 1970; Tinto, 1975; Ethington, 1990), quienes consideran a los factores familiares respecto al apoyo al logro, por medio de expectativas y de motivaciones, además de fomentar los factores económicos para la trayectoria del estudiante.

Sobre la relación entre los factores académico-educativos, existen efectos tanto positivos como negativos. Así lo evidencia la relación positiva que existe entre los antecedentes familiares y los ingresos familiares con la dependencia del establecimiento de origen de los estudiantes, es decir, si aumenta el valor asociado a los antecedentes familiares y aumentan los ingresos, el estudiante tendrá mayor posibilidad de acceder a un colegio particular pagado, lo que a su vez tendrá influencia positiva en el tipo de universidad a la cual ingresará posteriormente el estudiante. 
A su vez, la clasificación de las instituciones de educación superior tiene un efecto negativo en la retención, es decir, mientras se está en una universidad de investigación y doctorado (la más alta clasificación) más difícil es la retención. En el contexto universitario se debiera considerar que estas universidades tienen estándares más altos de exigencia académica, más aún cuando se evalúa la relación retención/deserción al interior de estas universidades, la retención es proporcionalmente mayor que en otras instituciones de menor calificación, pero que reciben mayor cantidad de estudiantes (SIES, 2015).

En el caso de las variables de control sexo y edad, se observó que las mujeres tienen mayores posibilidades de permanecer en la educación superior que los hombres. Sobre la edad, el parámetro estimado es débil y negativo, en donde a mayor edad al matricularse, menor tendencia a estar retenido en educación superior. Estos resultados son similares a lo obtenido en otros estudios, tales como Barrios (2013) y Bordón, Canals y Rojas (2015).

Las variables relacionadas con las capacidades y habilidades de los estudiantes fueron consideradas (PSU y NEM), pero no fueron estadísticamente significativas para el modelo, pues no permitieron un buen ajuste por el bajo efecto sobre retención. No obstante, cabe señalar que poseen un efecto implícito para el acceso a determinadas universidades, por lo que su presencia podría estar contenida en el efecto de la clasificación de las instituciones. En ese sentido, es necesario señalar que existe un conjunto de variables no visibles en el modelo y que conforman lo no explicado por éste.

Respecto de los modelos por áreas del conocimiento, se observan diferencias interesantes entre los estudiantes que cursan programas en educación y salud, siendo los primeros programas con menor selectividad, en donde los factores familiares y económicos disminuyen su preponderancia. En cambio, en el caso del área de salud, con programas con mayores niveles de selectividad, los factores familiares y económicos son más relevantes.

A partir de estos resultados obtenidos, es posible contextualizar el problema con la realidad existente en el sistema educativo chileno, en donde existe una fuerte segregación de jóvenes y estudiantes a partir de sus antecedentes familiares y económicos (Canales, 2016; Orellana, 2011), esto considerando el acceso, permanencia y titulación. En ese sentido, el sistema educativo chileno contribuye a la reproducción de diferencias sociales que parecieran estar más bien en un nivel estructural. Por lo mismo, pensar en programas y/o intervenciones aplicadas para fomentar la permanencia de los estudiantes en educación superior debe considerar ciertos elementos culturales del país que operan en una lógica de desigualdad social e institucional (Araujo, 2016). 
Para finalizar, es necesario mencionar que el modelo propuesto es susceptible a cambios, la incorporación de variables subjetivas asociadas a la medición de expectativas, de motivaciones, de intereses, entre otros, resulta fundamental para continuar con el estudio de la deserción y la retención en la educación superior chilena.

\section{Referencias}

Acuña Véliz, C. A. (2012). Acceso y Deserción en la Educación Superior, caso aplicado a Chile. Santiago: Tesis para optar al grado de Magíster en Economía Aplicada, Universidad de Chile.

Araujo, K. (2016). La calle y las desigualdades interaccionales. Santiago: PNUD, Serie Documentos de Trabajo.

Atencio Abarca, I. y López, M. N. (2016a). Empleabilidad e ingresos en carreras del área de la salud. Santiago: Ministerio de Educación, Servicio de Información de Educación Superior.

Atencio Abarca, I. y López, M. N. (2016b). Empleabilidad e ingresos en carreras de educación. Santiago: Ministerio de Educación, Servicio de Información de Educación Superior.

Barrios, A. (2013). Deserción universitaria en Chile: Incidencia del financiamiento y otros factores asociados. Revista del Centro de Investigación Social de Un Techo para Chile, 59-72.

Bean, J. P. (1980). Dropouts and Turnover: The Synthesis and Test of a Causal Model of Student Attrition. Research in Higher Education, Vol. 12, No. 2, 155-187.

Bean, J. P. (1985). Interaction Effects Based on Class Level in an Explanatory Model of College Student Dropout Syndrome. American Educational Research Journal, Vol. 22, No. 1, 35-64.

Belloc, F., Maruotti, A. y Petrella, L. (2010). University drop-out: an Italian experience. Higher Education, Volume 60, Issue 2, 127-138.

Bollen, K. A. (1989). Structural Equations with Latent Variables. New York: John Wiley \& Sons.

Bordón, P., Canals, C. y Rojas, S. (2015). Retención en los programas e instituciones de educación superior. Nueva evidencia para Chile. Estudios de Política Educativa, No. 2, 174-201.

Brown, T. A. (2006). Confirmatory Factor Analysis for Applied Research. New York: The Guilford Press.

Canales, A. (2016). Diferencias Socioeconómicas en la postulación a las Universidades Chilenas: El rol de factores académicos y no académicos. Revista Calidad en la educación, n. 44, 129-157.

Centro de Estudios-Mineduc. (2012). Serie Evidencias: Deserción en la educación superior en Chile. Santiago: Ministerio de Educación.

DesJardins, S., Ahlburg, D. y McCall, B. (1999). An event history model of student departure. Economics of Education Review 18, 375-390. 
DFE (2015). Memoria Financiamiento Estudiantil 2014. Santiago: Ministerio de Educación.

Díaz Peralta, C. (2008). Modelo conceptual para la deserción estudiantil universitaria chilena. Estudios Pedagógicos XXXIV, No 2, 65-86.

Díaz, M. Á., Pardo Merino, A. y San Martín Castellanos, R. (2010). Modelos de ecuaciones estructurales. Papeles del psicólogo, 31 (1), 34-45.

Donoso, S. y Schiefelbein, E. (2007). Análisis de los modelos explicativos de retención de estudiantes en la Universidad: Una visión desde la desigualdad social. Estudios Pedagógicos, vol. xxxiII, núm. 1, 7-27.

Duque, L. C., Duque, J. C. y Suriñach, J. (2013). Learning outcomes and dropout intentions: an analytical model for Spanish universities. Educational Studies, 39, 261-284.

Durkheim, E. (1989). Suicide: a study in sociology. London: Routledge and Kegan Paul.

Eccles, J., Adler, T. F., Futterman, R., Goff, S. B., Kaczala, C. M., Meece, J. L. y Midgley, C. (1983). Expectancies, values, and academic behaviors. En J. T. Spence, Achievement and Achievement Motives: Psychological and Sociological Approaches (págs. 75-146). San Francisco: W. H. Freeman and Company.

Espinoza, O. y González, L. E. (2013). Access to higher education in Chile: A public vs. private analysis. Prospects 43, 199-214.

Ethington, C. A. (1990). A psychological model of student persistence. Research in Higher Education, Vol. 31, No. 3, 266-269.

Fishbein, M. y Ajzen, I. (1975). Belief, attitude, intention and behavior: An Introduction to Theory and Research. EE.UU.: Addison-Wesley.

Fonseca, G. y García, F. (2016). Permanencia y abandono de estudios en estudiantes universitarios: un análisis desde la teoría organizacional. Revista de la Educación Superior 45 (179), 25-39.

Forsman, J., Linder, C., Moll, R., Fraser, D. y Andersson, S. (2012). A new approach to modelling student retention through an application of complexity thinking. Studies in Higher Education, 1-19.

Himmel, E. (2002). Modelos de análisis para la deserción estudiantil en la educación superior. Revista Calidad en la Educación, No 17, 91-107.

Ishitani, T. T. y DesJardins, S. L. (2002). Longitudinal Investigation of Dropout from College in the United States. Paper presented at the Annual Meeting of the Association for Institutional Research, 42nd, Toronto, Ontario, Canada, 34 .

Manzi Astudillo, J. (2011). ¿Qué características de la formación inicial de los docentes se asocian a mayores avances en su aprendizaje de conocimientos disciplinarios? Santiago: FONIDE.

Meece, J. L., Eccles Parsons, J. E., Kaczala, C. M., Goff, S. B. y Futterman, R. (1982). Sex differences in math achievement: Toward a model of academic choice. Psychological Bulletin, Vol. 91, No. 2, 324-348.

Meller, P. (2010). Carreras Universitarias, rentabilidad, selectividad y discriminación. Santiago: Uqbar.

Montmarquette, C., Mahseredjian, S. y Houle, R. (2001). The determinants of university dropouts: a bivariate probability model with sample selection. Economics of Education Review 20, 475-484. 
Orellana, V. (2011). Nuevos estudiantes y tendencias emergentes en la educación superior. Una mirada al Chile del mañana. Santiago: Aequalis.

Pearson. (2013). Final Report. Evaluation of the Chile PSU. Pearson.

Price, J. L. (1977). The Study of Turnover. Ames: Iowa State University Press.

Reyes, C. y Rosso, P. P. (2013). Una nueva clasificación de las universidades chilenas. En M. V. Santelices, J. J. Ugarte y J. Salmi, Clasificación de Instituciones de Educación Superior (págs. 135-151). Santiago: Ministerio de Educación.

Santelices, V., Catalán, X., Horn, C. y Kruger, D. (2013). Determinantes de Deserción en la Educación Superior Chilena, con Énfasis en Efecto de Becas y Créditos. Santiago: FONIDE-Mineduc.

SIES (2015). Informe de retención de primer año de las cohortes 2010-2014. Santiago: Ministerio de Educación.

Skrondal, A. y Rabe-Hesketh, S. (2005). Structural Equation Modeling: Categorical Variables. John Wiley \& Sons, Ltd.

Smith, J. P. y Naylor, R. A. (2001). Dropping out of university: A statistical analysis of the probability of withdrawal for UK university students. Journal of the Royal Statistical Society: Series A (Statistics in Society), Volume 164, Issue 2, 389-405.

Spady, W. G. (1970). Dropouts from higher education: An interdisciplinary review and synthesis. Interchange 1 (1), 64-85.

Tinto, V. (1975). Dropout from Higher Education: A Theoretical Synthesis of Recent Research. Review of Educational Research, Vol. 45, No. 1, 89-125.

Tinto, V. (1988). Stages of Student Departure: Reflections on the Longitudinal Character of Student Leaving. The Journal of Higher Education Vol. 59, No. 4, 438-455.

Urzúa, S. (2012). La rentabilidad de la educación superior en Chile. ¿Educación superior para todos? Documento de trabajo No 386. Santiago: Centro de Estudios Públicos. 